\title{
Ictal postural phantom limb sensation is associated with impaired mental imagery of body parts
}

\author{
Lukas Heydrich $^{1,2,6}{ }^{(1)} \cdot$ Mariia Kaliuzhna $^{1} \cdot$ Sebastian Dieguez $^{1,4}$, \\ Roger Nançoz ${ }^{3}$ - Olaf Blanke ${ }^{1,2,5} \cdot$ Margitta Seeck $^{2}$
}

Dear Sirs,

The scientific study of neurological patients suffering from alterations in perceptual bodily experience such as the experience of body part displacement [1], the delusional misidentification of one's own body part [2], as well as the illusory perception of the entire body [3], has revealed the presence of brain structures overseeing the integration of multisensory bodily signals for body awareness [4].

Postural phantom limb sensation refers to the impression that one's limb is not in the correct physical position, e.g., the dissociation between the perceived position of an intact body part and its actual position [5]. It has been described in the context of severe sensori-motor deficits following stroke in the parietal lobe of the right hemisphere of the brain, but also in patients with subcortical brain damage [5].

\section{Electronic supplementary material}

Lukas Heydrich

lukas.heydrich@insel.ch

1 Laboratory of Cognitive Neuroscience, Brain Mind Institute, École Polytechnique Fédérale de Lausanne, Lausanne, Switzerland

2 Department of Neurology, University Hospital Geneva, Geneva, Switzerland

3 Avenue du Rothorn 20, 3960 Sierre, Switzerland

4 Department of Medicine, Neurology, University of Fribourg, Fribourg, Switzerland

5 Center for Neuroprosthetics, École Polytechnique Fédérale de Lausanne, Lausanne, Switzerland

6 Department of Neurology, Inselspital, University Hospital and University of Bern, Bern, Switzerland
Here, we describe a patient reporting an ictal postural phantom limb sensation of her right hand due to a dysplasia of the left temporo-parietal junction. We further demonstrate that the patient suffers from impaired mental imagery of body parts and discuss ictal postural limb sensations with respect to involved brain mechanisms and regions.

Patient $\mathrm{C}$ is a 25 -year-old, right-handed woman suffering from complex partial seizures with secondary generalization. The seizures are characterized by the stereotypic impression "that her right hand does no longer belong to her" while "being in a strange and physiologically impossible position and angle with respect to the rest of the her body". The experience was non-visual (the patient was too frightened to look at the hand) and no voluntary or involuntary movement of the phantom was reported. In addition, the phantom was experienced as replacing the actual hand. This was associated with motor aphasia, head deviation to the right, and followed by secondary tonicclonic generalization. The average seizure frequency was one seizure per year under antiepileptic treatment (lamotrigine, clonazepam).

Several interictal electroencephalogram (EEG) recordings demonstrated intermittent focal slowing and sharp waves over the left parietal cortex without epileptiform activity (see Fig. 1a). A high-resolution MRI revealed a dysplasia in the left inferior parietal lobule and the left superior temporal gyrus, e.g., the left temporo-parietal junction (see Fig. 1b). Extensive interictal neuropsychological testing and a thorough neurological exam were normal. A psychiatric evaluation revealed a severe anxiety disorder secondary to the epileptic seizures.

We compared the patient's performance in a mental rotation task involving body parts (i.e., the right arm; body condition) with the performance using a non-corporeal external object (i.e., the letter "F"; object condition). Both 
A

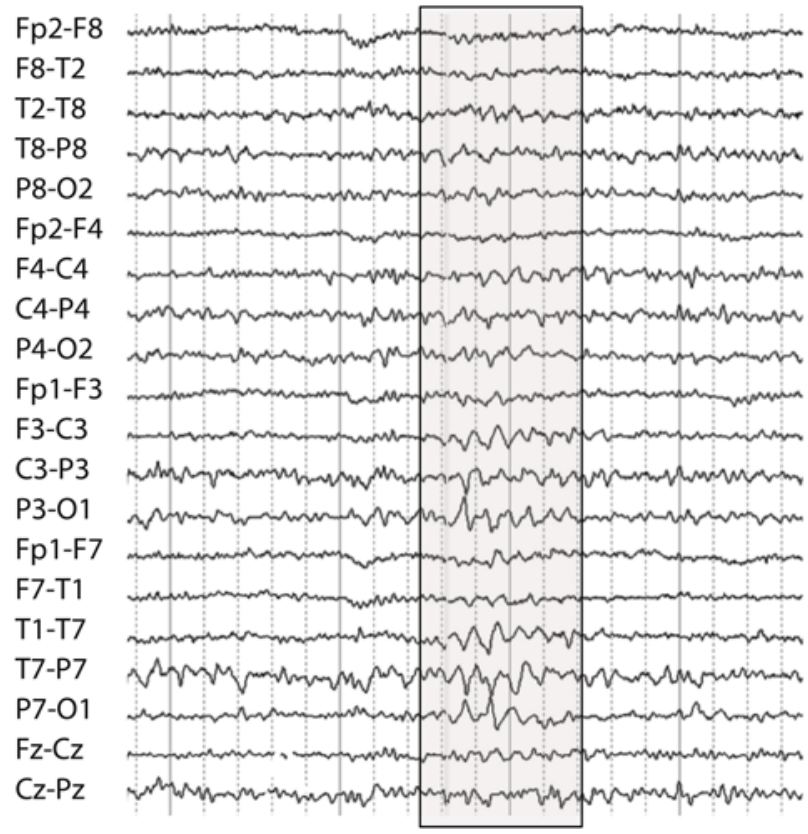

B

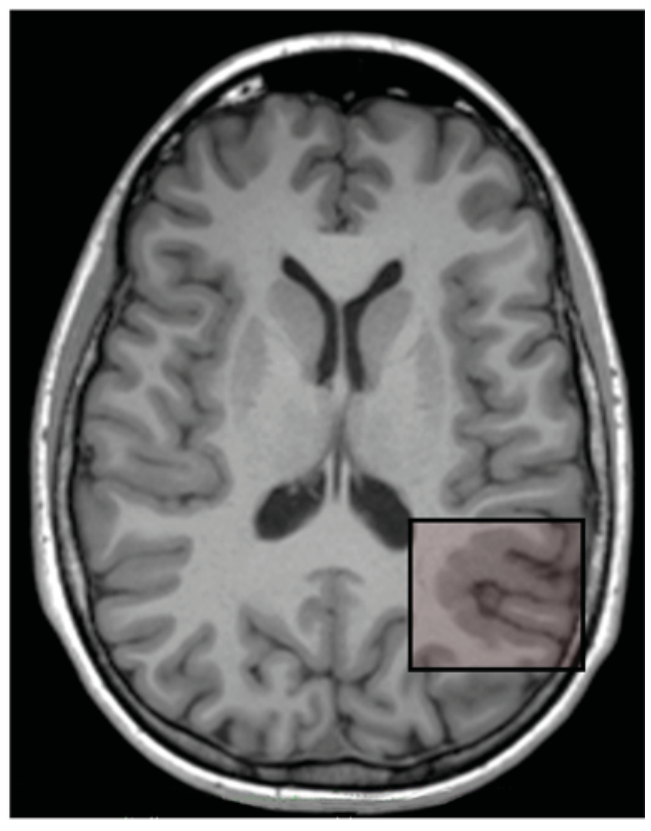

Fig. 1 a Interictal EEGs demonstrated an intermittent focal slowing and sharp waves over the left parietal cortex (P3, P7) without epileptiform activity. A bipolar longitudinal montage is used. b MRI

types of stimuli were presented at five different angles $\left(0^{\circ}-\right.$ $120^{\circ}$ ) and were either in a normal view or in an inverse view (see Fig. 2). The patient had to determine as quickly as possible whether the stimulus was presented in the correct or the inverse view. We predicted reaction times (RTs) to be longer as a function of degree of the deviation from normal upright view, i.e., higher deviations resulting in longer RTs (the so-called mental rotation function; for details, refer to Refs. [6, 7]). The behavioural data of the patient were compared to a control group of eight healthy subjects [for further details and the statistical analysis, refer to the electronic supplementary material (ESM)].

An ANOVA with factors task (body, object) and angle $\left(0^{\circ}, 30^{\circ}, 60^{\circ}, 90^{\circ}, 120^{\circ}\right)$ showed that control subjects were generally slower in the body condition as compared to the object condition (main effect of task, $F=37,73$, $p<0.001$, see Fig. 2). Importantly, as predicted, the effect of angle was present in both the body condition and the object condition (main effect of angle, $F=17, p<0.001$ ). Post hoc analysis showed that the effect of angle was present in the object condition both for the patient and the control subjects $(p=0.12)$, while no effect of angle was found in the patient when carrying out the body condition ( $p<0.001$; for further details, refer to ESM and Fig. 2).

Although postural phantoms resulting from cerebral lesions are considered a rare phenomenon, a recent study showed that up to $50 \%$ of stroke patients suffered from postural phantom limb sensations after a severe sensori- (axial plane, T1-weighted sequence) reveals a dysplasia in the left inferior parietal lobule and the left superior temporal gyrus (indicated by the red area)

motor deficit [5]. During the sensation of a postural phantom, the patient has the impression of his arm (or leg) being in a position, which is not in accordance with its actual physical position, indicating a lack of proprioceptive processing. Accordingly postural phantoms are mostly a non-visual sensation and stronger while being deprived from visual input, e.g., during the night. Our patient also reported the non-visual sensation of her arm being in a strange and anatomically impossible position with respect to the rest of her body while disowning her right hand at the same time. This link between proprioception and body ownership has been studied recently in healthy participants, showing that interference with proprioceptive processing [8] and proprioceptive-visual integration [9] results in a change in body ownership and body schema.

Interestingly, in contrast to the healthy control group and a control condition using an object, our patient did not show the so-called mental rotation function for body parts (e.g., the dependency of RTs on the angle of the displayed stimulus), which reflects the process of the participants imagining their own right hand in the orientation of the stimulus to judge the anatomical plausibility of the visual stimulus [7, 10].

It has previously been shown that the mental imagery of body parts including a mental transformation task mostly activates the left parietal lobe [10,11], and that damage to the left parietal cortex, as seen in our patient, results in impaired mental imagery of the contralesional limb [7], 
Fig. 2 Task and results. Participants had to report as fast and as correctly as possible whether the stimulus was correct or mirror-reversed. The stimuli were presented in five different angles. a Illustration of the stimuli used and reaction times (RTs) of the patient and the control participants in the object condition. b Illustration of the stimuli used and RTs of the patient and the control participants in the body condition. Error bars represent standard error of mean
A

Object Condition
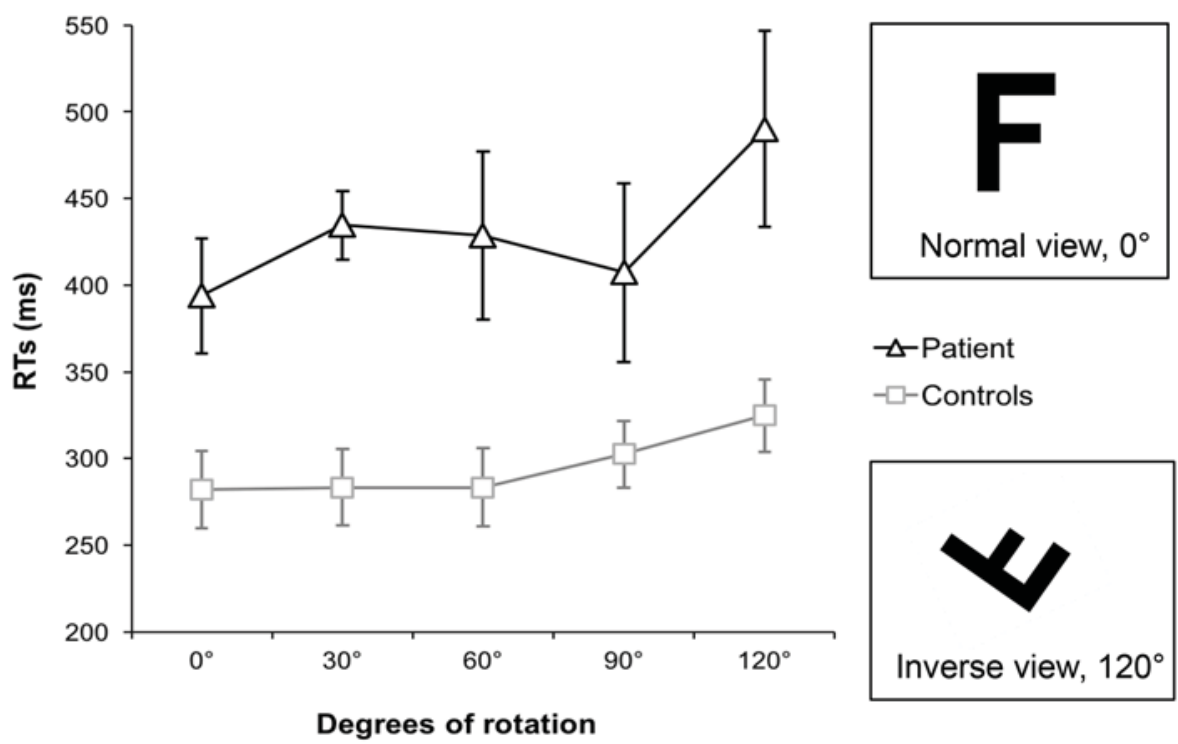

B

Body Condition

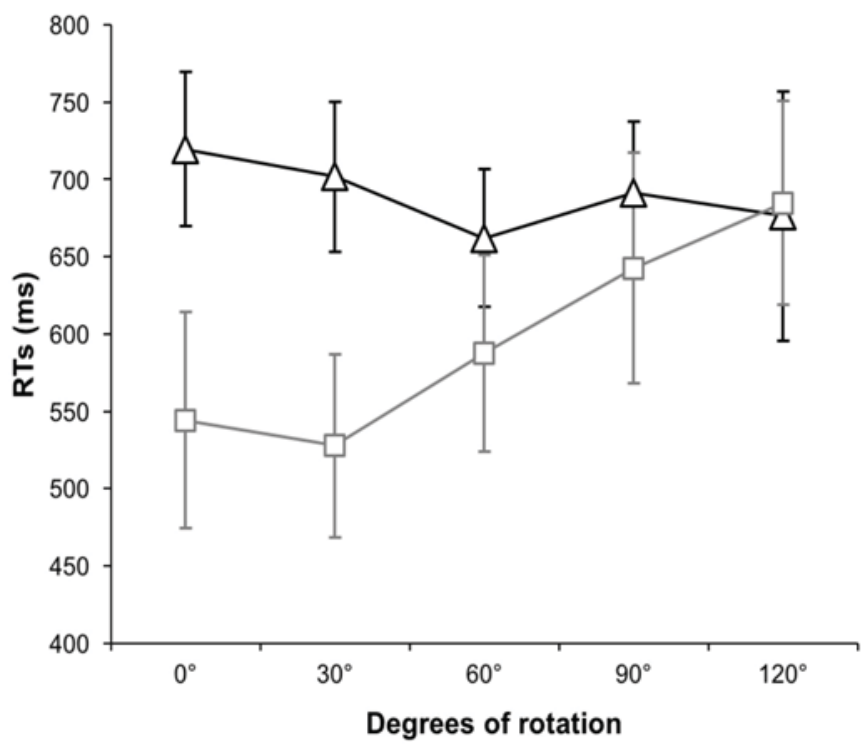

$-\triangle$-Patient

$-\square$-Controls

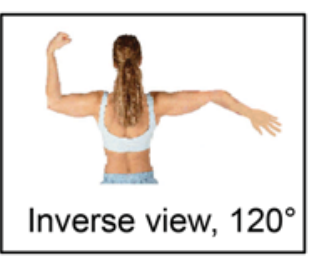

while mental transformation of external objects activates the parietal lobes of both hemispheres [12]. In addition, it has been suggested that the temporo-parietal junction serves as one of the core centres responsible for the integration of multisensory bodily signals and body awareness [3, 4].

Therefore, we conclude that the finding of a selective impairment of mental imagery of body parts in this patient suffering from a dysplasia of the left temporo-parietal junction not only relates to the patient's ictal sensation of her arm being in an anatomically implausible position, e.g., the postural phantom sensation, but also highlights the role of the left temporo-parietal junction in integrating multisensory information from the body and in the representation of the topological body map [13].

Acknowledgements This work is supported by the Swiss National Science Foundation (Grants 140332, 140338, 146633, 33CM30-124 089, and 51AU40-125759), the Cogito Foundation, the Roger de Spoelberch Foundation, and the Bertarelli Foundation. We would like to thank Dr. Andrea Rossetti for referring the patient for pre-surgical evaluation.

\section{Compliance with ethical standards}

Conflicts of interest The authors declare that they have no conflict of interest. 
Ethical standards The study was approved by the ethical committee of the University Hospital of Geneva and written informed consent was obtained from all participants, including the patient.

Informed consent Informed consent for the publication of the case report anonymously was obtained from the patient.

\section{References}

1. Nightingale S (1982) Somatoparaphrenia: a case report. Cortex 18:463-467

2. Gerstmann J (1942) Problem of imperception of disease and of impaired body territories with organic lesions. Relation to body schema and its disorders. Arch Neurol Psychiatry 48:890-913

3. Heydrich L, Blanke O (2013) Distinct illusory own-body perceptions caused by damage to posterior insula and extrastriate cortex. Brain 136:790-803

4. Ionta S, Heydrich L, Lenggenhager B et al (2011) Multisensory mechanisms in temporo-parietal cortex support self-location and first-person perspective. Neuron 70:363-374

5. Antoniello D, Kluger BM, Sahlein DH, Heilman KM (2010) Phantom limb after stroke: an underreported phenomenon. Cortex 46:1114-1122
6. Arzy S, Overney LS, Landis T, Blanke O (2006) Neural mechanisms of embodiment: asomatognosia due to premotor cortex damage. Arch Neurol 63:1022-1025

7. Overney LS, Blanke O (2009) Impaired imagery for upper limbs. Brain Topogr 22:27-43

8. Walsh LD, Moseley GL, Taylor JL, Gandevia SC (2011) Proprioceptive signals contribute to the sense of body ownership. J Physiol 12:3009-3021

9. Folegatti A, de Vignemont F, Pavani F et al (2009) Losing one's hand: visual-proprioceptive conflict affects touch perception. PLoS One 4:e6920

10. Petit LS, Harris IM (2005) Anatomical limitations in mental transformations of body parts. Vis Cogn 12:737-758

11. Bonda E, Petrides M, Frey S, Evans A (1995) Neural correlates of mental transformations of the body-in-space. Proc Natl Acad Sci USA 92:11180-11184

12. Kosslyn SM, DiGirolamo GJ, Thompson WL, Alpert NM (1998) Mental rotation of objects versus hands: neural mechanisms revealed by positron emission tomography. Psychophysiology $35: 151-161$

13. Corradi-Dell'acqua C, Ueno K, Ogawa A et al (2008) Effects of shifting perspective of the self: an fMRI study. Neuroimage 40:1902-1911 